\title{
Cathepsin D Expression in Oral Squamous Cell Carcinoma and Cancer-Associated Fibroblasts: A Preliminary Study
}

\author{
Dokyeong $\mathrm{Kim}^{1}$ and Sook Moon ${ }^{2, \dagger}$ \\ ${ }^{1}$ Precision Medicine Research Center, Department of Biomedicine \& Health Sciences, College of Medicine, The \\ Catholic University of Korea, Seoul 06591, '2Department of Dental Hygiene, Daejeon Health Institute of \\ Technology, Daejeon 34504, Korea
}

\begin{abstract}
Background: Cancer-associated fibroblasts (CAFs) are abundant in tumor microenvironments and interact with cancer cells to promote tumor proliferation in oral squamous cell carcinoma (OSCC). Cathepsin D (CTSD) is a soluble lysosomal aspartic endopeptidase involved in tumor proliferation and angiogenesis. In this preliminary study, we observed CTSD expression in OSCC and CAFs, postulating that CTSD might act as a bridge between OSCC and CAFs.

Methods: Human epidermal keratinocytes (HEKs), OSCC, and immortalized human normal oral fibroblasts (hTERT-hNOFs) were used in this study. Additionally, we used hTERT-hNOFs transfected with an empty vector, WT (wild-type)-YAP (Yes-associated protein), and YAPS127A (YAP serine 127 to alanine). YAP127A hTERT-hNOFs activated fibroblasts similar to CAFs. To identify CTSD expression between OSCC and CAFs, conditioned medium (CM) was collected from each cell. Protein expression of CTSD was identified by western blotting.

Results: To identify the expression of CTSD in fibroblasts stimulated by OSCC, we treated fibroblasts with CM from HEK and OSCC. Results indicated that hTERT-hNOFs with OSCC CM showed a weakly increased expression of CTSD compared to stimulation by HEK CM. This indicates that CAFs, YAPS127 hTRET-hNOFs, overexpress CTSD protein. HEK cells showed no CTSD expression, regardless of treatment with fibroblast CM, whereas OSCC highly expressed CTSD proteins compared with the CTSD expression in HEK cells. We also found that CTSD expression was unaffected by changes in transforming growth factor- $\beta$ levels.

Conclusion: This study proposes that CTSD might have potential as an interacting executor between OSCC and CAFs. Further studies are needed to investigate the role of CTSD in tumor and stromal cells.
\end{abstract}

Key Words: Cathepsin D, Fibroblasts, Oral squamous cell carcinoma

\section{Introduction}

Tumors contain diverse cell types, including endothelial cells, pericytes, immune cells, and fibroblasts. The interaction between tumors and their surrounding cells can influence tumor growth, invasion, and metastasis ${ }^{1,2)}$. Cancer-associated fibroblasts (CAFs) are abundant in tumor microenvironments and interact with cancer cells to promote tumor proliferation ${ }^{3,4)}$. The secretion of growth factors, chemokines, and enzymatic mediators such as transforming growth factor- $\beta$ (TGF- $\beta)^{5)}$, vascular endothelial growth factor $^{6)}$, interleukin- $1 \alpha^{7)}$, and matrix metalloproteinases ${ }^{8)}$ can remodel the extracellular matrix and induce oncogenic signals, promoting cancer growth and metastasis. Despite multiple studies, tumor-stroma interactions remain unclear and require the investigation of oral squamous cell carcinoma (OSCC), a relatively rare cancer.

The cathepsin family, cathepsin B, G, D, K, L, and V, are well-known lysosomal proteases that play a role in protein degradation, cellular stress signaling, and inflammation ${ }^{9}$. Cathepsin D (CTSD) is a soluble lysosomal aspartic endopeptidase involved in tumor proliferation and 
angiogenesis ${ }^{10)}$. CTSD is considered a prognostic marker, and its overexpression is associated with breast cancer recurrence and prognosis ${ }^{10,11)}$.

This preliminary study observed CTSD expression in OSCC and CAFs (called the activated fibroblast phenotype) and postulated that CTSD might act as a bridge between OSCC and CAFs.

\section{Materials and Methods}

\section{Reagents}

Reagents included antibodies against CTSD (Santa Cruz Inc., Dallas, TX, USA), $\beta$-actin (Bioworld, Irving, TX, USA), and horseradish peroxidase-conjugated antimouse or rabbit secondary antibodies (Cell Signaling Technology, Beverly, MA, USA). TGF- $\beta 1$ (Merck KGaA, Darmstadt, Germany) was used to activate fibroblasts in the empty vector; wild-type (WT)-YAP (Yes-associated protein) and YAPS127A (YAP serine 127 to alanine) were transfected to immortalized human normal oral fibroblasts (hTERT-hNOFs).

\section{Cell cultures}

We cultured human epidermal keratinocytes (HEKs), YD10B OSCC cells, and hTERT-hNOFs, selecting representative cells for each type. HEK cells were used as normal keratinocytes for comparison with OSCC cells. YD10B cells, derived from the tongue of OSCC patients, were used as OSCC cells. Additionally, hTERT-hNOFs, obtained by hTERT transfection, were used to reproduce the in vitro fibroblast study results. The cells were obtained from the Department of Oral Pathology, Yonsei University College of Dentistry (Seoul, Korea). The hTERT-hNOFs were grown in F medium, consisting of Dulbecco's modified Eagle's medium (DMEM; Gibco BRL, Gaithersburg, MD, USA) and Ham's Nutrient Mixture-F12 (Gibco BRL) in a ratio of 3:1, supplemented with $10 \%$ fetal bovine serum and $1 \%$ penicillin/ streptomycin. HEKs were maintained in keratinocyte growth medium (Lonza, Basel, Switzerland) and used for eight passages. YD10B OSCC cells were grown in EF medium, a mixture of $\mathrm{F}$ and $\mathrm{E}$ media (ratio, 9:1). The $\mathrm{E}$ medium had $0.01 \mu \mathrm{g} / \mathrm{ml}$ of cholera toxin, $0.04 \mu \mathrm{g} / \mathrm{ml}$ of hydrocortisone, $0.5 \mu \mathrm{g} / \mathrm{ml}$ of insulin, $0.5 \mu \mathrm{g} / \mathrm{ml}$ of apotransferrin, and $0.2 \mu \mathrm{g} / \mathrm{ml}$ of 3'-5-triiodo-1-thyronine from Sigma Aldrich (St. Louis, MO, USA). To identify the CTSD expression, we used three types of fibroblasts that established by transfecting pcDNA4/HisMax B mammalian expression vector (Invitrogen, Waltham, MA, USA), pcDNA4/HisMaxB-YAP1 (Addgene, Cambridge, MA, UK), and pcDNA4/HisMaxb-YAP-S127A (Addgene), as previously described ${ }^{12)}$. All cells were maintained in an incubator at $37^{\circ} \mathrm{C}$ and $5 \% \mathrm{CO}_{2}$. The cell culture medium was changed every three days.

\section{Collection of conditioned medium}

The CM was collected from each cell (at $70 \%$ confluence in 100-mm dishes) and incubated in a serum-free medium (DMEM:F12 in a 3:1 ratio) for 48 hours. The supernatants were centrifuged at $400 \mathrm{~g}$ for 5 minutes and collected. The $\mathrm{CM}$ was stored at $-80^{\circ} \mathrm{C}$ before the experiments.

\section{Protein lysis and Western blot}

The cells were washed with phosphate-buffered saline (PBS) at $80 \%$ confluence and then lysed using cell lysis buffer (Cell Signaling Technology). Protein lysates were incubated for 30 minutes on ice and vortexed every 5 minutes. After 30 minutes, the lysates were centrifuged at $>15,000 \mathrm{rpm}$ for 10 minutes at $4^{\circ} \mathrm{C}$ and then boiled for 5 minutes at 95 to $100^{\circ} \mathrm{C}$ in sodium dodecyl sulfate (SDS) sample buffer. The protein samples were separated using SDS-polyacrylamide gel electrophoresis and transferred to a polyvinylidene difluoride membrane (Cyvita, Malborough, MA, USA). The blots were blocked using $5 \%$ skim milk in PBS with Tween 20, incubated with the appropriate primary antibodies, and detected using chemiluminescence (GenDEPOT, Katy, TX, USA).

\section{Results}

\section{Cathepsin D expression in activated fibroblasts}

To identify the CTSD expression in fibroblasts stimulated by OSCC, we treated them with CM from HEK and YD10B cells. The hTERT-hNOFs stimulated with YD10B CM showed weakly increased CTSD expression 
(1.11-fold increase) compared to cells stimulated with HEK CM (Fig. 1A). As YAP127A fibroblasts activated fibroblasts such as CAFs ${ }^{12,13)}$, we examined the expression of CTSD in each fibroblast: the empty vector, WT-YAP1-, and YAPS127A-transfected hTERT-hNOFs (Fig. 1B). YAPS127A hTERT-hNOFs showed a 1.31- and 1.24-fold increase in CTSD expression compared to the empty vector and WT-YAP1 hTERT-hNOFs, respectively. This indicates that CAFs, with their ability to remodel the matrix and maintain nuclear YAP activity in YAP127A hTERT-hNOFs ${ }^{13)}$, overexpress the CTSD protein. We also determined CTSD expression in the absence or presence of TGF- $\beta$ and found no effect on TGF- $\beta$ levels.

Altogether, we identified the CTSD expression in activated fibroblasts, such as those stimulated by CM of OSCC cells that maintained nuclear YAP activity in YAPS127A hTERT-hNOFs.

\section{Cathepsin $D$ expression in oral squamous cell carcinoma}

Next, we examined the expression of CTSD in the neoplastic epithelial cells. To mimic indirect stimulation of epithelial cells by fibroblasts, CM was collected from hTERT-hNOFs and applied to HEK and YD10B cells. HEK cells weakly expressed CTSD with and without CM application, whereas YD10B cells strongly expressed the CTSD protein with a 1.41 -fold increase compared with the CTSD expression in HEK cells without CM. After treatment with CM from hTERT-hNOFs, YD10B cells showed a 1.57-fold increase compared to HEK-treated CM from hTERT-hNOFs. In conclusion, OSCC cells expressed more CTSD than normal keratinocytes.

\section{Discussion}

The interactions between cancer cells and stromal fibroblasts play a critical role in carcinogenesis ${ }^{2}$. One of the ways stromal cells regulate epithelial cell function is
$\mathbf{A}$
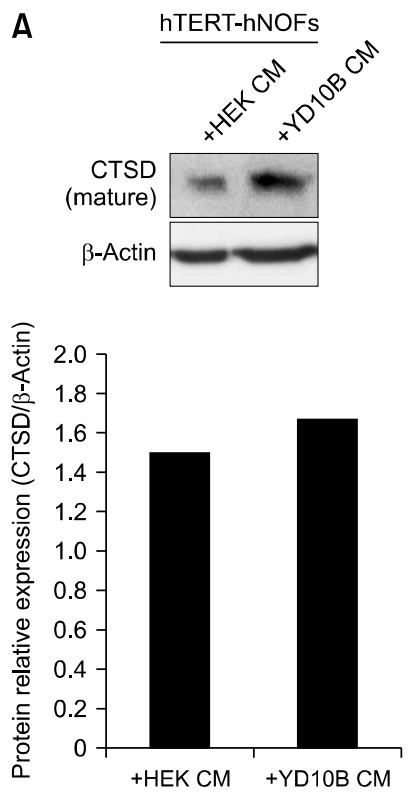

B
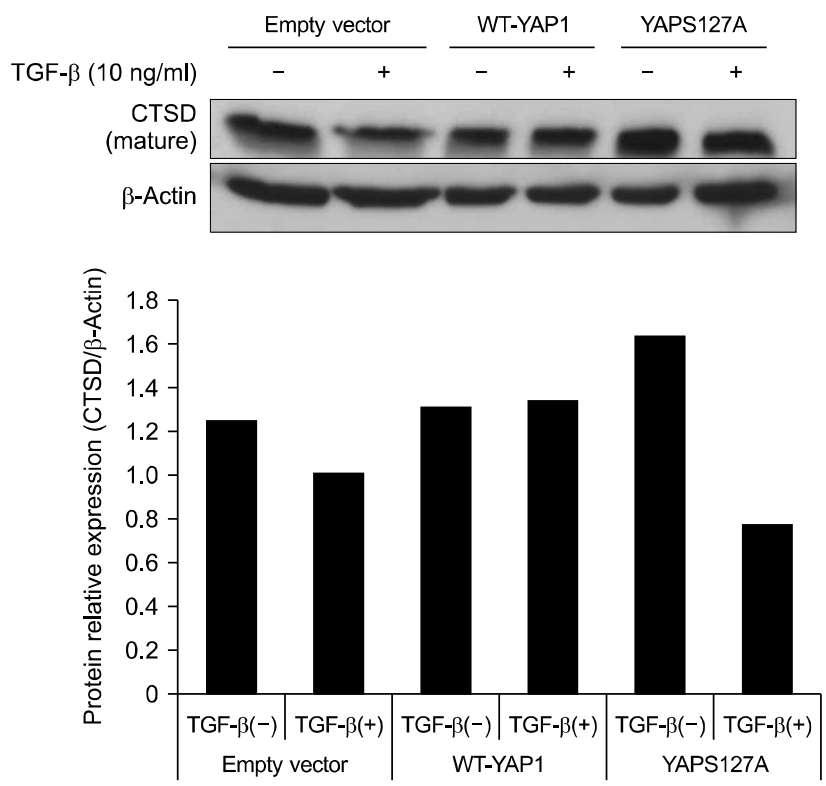

Fig. 1. Protein expression of CTSD in activated fibroblasts. (A) hTERT-hNOFs were applied with CM from HEK and YD10B cells. HEK and YD10B cells were grown in serum-free media comprising DMEM and F12 at a 9:1 ratio for 48 hours. The bar graph quantifies each band intensity (Image J software); the CTSD protein expression was normalized to $\beta$-Actin. (B) Fibroblasts were lysed with or without TGF- $\beta 10 \mathrm{ng} / \mathrm{ml}$ for 24 hours in serum-free medium, and the protein samples were loaded. Empty vector: pcDNA4/HisMaxB mammalian expression vector transfected hTERT-hNOFs. WT-YAP1: pcDNA4/HisMaxB-WT YAP1 transfected hTERT-hNOFs. YAPS127A: pcDNA4/ HisMaxB-YAPS127A transfected hTERT-hNOFs. CTSD: Cathepsin D, hTERT-hNOFs: immortalized human normal oral fibroblasts, CM: conditioned medium, HEK: human epidermal keratinocyte, DMEM: Dulbecco's modified Eagle's medium, TGF- $\beta$ : transforming growth factor- $\beta$, WT: wild-type, YAP: Yes-associated protein, YAPS127A: YAP serine 127 to alanine. 
through the secretion of growth factors. TGF- $\beta$, commonly secreted by fibroblasts, can either suppress or promote tumorigenesis ${ }^{14)}$. TGF- $\beta$ belongs to a superfamily of cytokines that act on protein kinase receptors at the plasma membrane to induce a plethora of biological signals, regulating cell growth and death, differentiation, immune response, angiogenesis, and inflammation ${ }^{5)}$. This study investigated CTSD expression in fibroblasts and OSCC cells. We first treated fibroblasts with CM from HEK and YD10B OSCC cells. The YD10B CM-treated hTERThNOFs showed a weak increase in CTSD expression compared to those stimulated by HEK CM (Fig. 1A). These results suggest that the expression of CTSD increases because of the interactions between OSCC and fibroblasts. Cathepsins are well-known lysosomal proteases that contribute to many cellular processes, including protein degradation and cellular stress signaling, cancer progression, invasion, metastasis, and inflammation. The cathepsin activity is crucial for inducing a reactive and supportive tumor stroma, making it a therapeutic target and a potent effector in wound repair response ${ }^{9)}$. In addition, cathepsins can be degraded during TGF- $\beta$ induced epithelial to mesenchymal transition in breast cancer cells ${ }^{15)}$. Among cathepsins, CTSD, a soluble lysosomal aspartic endopeptidase, is involved in tumor proliferation and angiogenesis ${ }^{10)}$. CTSD is a prognostic marker, and its overexpression is associated with breast cancer recurrence and prognosis ${ }^{10,11)}$. The presence of CTSD in stromal cells may influence the aggressive behavior of tumors, affect the concentration of this enzyme in tumor homogenates, and consequently alter its prognostic significance ${ }^{11)}$. Our results are similar to those of a previous report, indicating that CTSD-overexpressing tumor fibroblasts could assist cancer cells by remodeling the extracellular matrix ${ }^{16}$. Accordingly, we measured the expression of CTSD in several fibroblast phenotypes, including the empty vector, WT-YAP1-, and YAPS127Atransfected hTERT-hNOFs. We previously showed that YAPS127A-transfected hTERT-hNOFs can maintain nuclear YAP activity and enhance the tumor-promoting microenvironment ${ }^{12}$. In addition to the previous paper, YAPS127A fibroblasts have also been considered $\mathrm{CAFs}^{13)}$. Thus, we established fibroblast cell lines by transfecting
YAPS127 to reproduce the CAF-like phenotype in hTERT-hNOFs. Empty vector and WT-YAP1 were used as control groups for comparison with YAPS127A hTERT-hNOFs. Based on the previous results, we investigated the protein expression of CTSD in YAP activitymaintained fibroblasts (YAPS127A-transfected hTERT-hNOFs) as CAF-like phenotypical fibroblasts. In this study, CTSD expression was the highest in YAPS127A-transfected hTERT-hNOFs, as these fibroblasts were reactive and had CAF characteristics. Moreover, TGF- $\beta$ activates fibroblast differentiation to myofibroblasts, a well-known CAF type. However, there was no tendency with or without TGF- $\beta$ levels in the fibroblasts. Although TGF- $\beta$ can activate fibroblasts, CTSD expression decreased in TGF- $\beta$-treated YAPS127A fibroblasts compared to that in the TGF$\beta$-untreated fibroblasts. We hypothesized that this might be due to the diverse intercellular signaling pathways derived from TGF- $\beta$. Next, we measured CTSD expression in CM collected from hTERT-hNOFs, treated and

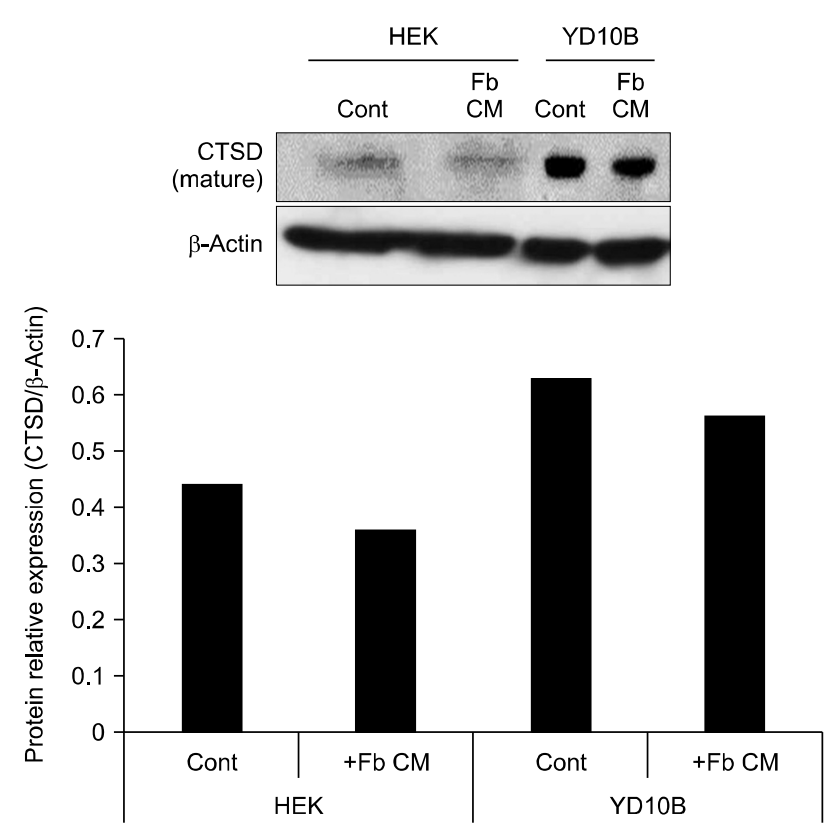

Fig. 2. Protein expression of CTSD in normal keratinocytes and oral squamous cell carcinoma. HEK and YD10B cells applied with CM from hTERT-hNOFs grown in serum-free media for 48 hours. The control is used only for serum-free medium without growing fibroblasts in HEK or YD10B cells. The bar graph quantifies each band intensity (Image $\mathrm{J}$ software); the CTSD protein expression was relative to $\beta$-Actin. CTSD: Cathepsin D, HEK: human epidermal keratinocyte, CM: conditioned medium, hTERT-hNOFs: immortalized human normal oral fibroblasts, $\mathrm{Fb} C M$ : the $\mathrm{CM}$ from hTERT-hNOFs. 
untreated HEK, and OSCC cells. CTSD protein levels were higher in untreated OSCC than in HEK cells (Fig. 2). OSCC may contain diverse growth factors, insulin-like growth factor-1, TGF, and epidermal growth factor, which can induce CTSD overexpression ${ }^{17)}$. In addition to untreated cells, OSCC showed a weak decrease in CTSD expression in both OSCC and HEK cells after treatment with $\mathrm{CM}$ from fibroblasts. This could be due to the secretion of pro-CTSD, rather than mature CTSD, by CM-stimulated fibroblasts. Further studies are needed to investigate the role of reciprocal CTSD interactions between tumors and stromal cells. Altogether, CTSD was expressed more in OSCC cells than in HEK cells. Furthermore, CTSD was highly expressed in the CAFs.

Dental hygienists help improve oral health through the care and prevention of oral diseases. Dental professionals, including dental hygienists, play an important role in distinguishing oral cancer from other oral diseases and require basic knowledge of oral cancer development and progression. Thus, this study could provide them with knowledge of oral cancer development. Nevertheless, our study has some limitations. First, it is insufficient to perform a statistical analysis because of the limited number of experiments. Second, our hypotheses cannot be generalized as we selected and used only one cell (HEK, YD10B, and hTERT-hNOFs) of each type, such as normal keratinocytes, OSCC cells, and fibroblasts. To overcome these limitations, replicate experiments should be performed with two or more cells from each cell type, such as normal keratinocytes, OSCC cells, and fibroblasts. This preliminary study to confirm the involvement of CTSD in the interactions between neoplastic epithelial cells and stromal cells during oral cancer development provides the basic infrastructure for further studies needed to investigate the role of CTSD in tumor cells and stromal cells in depth.

\section{Notes}

\section{Conflict of interest}

No potential conflict of interest relevant to this article was reported.

\section{Ethical approval}

All cell lines used were obtained from Yonsei University in the present study required ethics approval (IRB-2-2009-0002) for their use.

\section{Author contributions}

Conceptualization: Dokyeong Kim. Data acquisition: Dokyeong Kim. Formal analysis: Dokyeong Kim and Sook Moon. Funding: Dokyeong Kim. Supervision: Sook Moon. Writing-original draft: Dokyeong Kim and Sook Moon. Writing-review \& editing: Sook Moon.

\section{ORCID}

Dokyeong Kim, https://orcid.org/0000-0002-5154-9613

Sook Moon, https://orcid.org/0000-0002-5491-9448

\section{Acknowledgements}

This study was supported by research fund from Basic Science Research Program through the National Research Foundation of Korea (NRF) funded by the Ministry of Education (2021R1I1A1A01045571).

\section{References}

1. Baghban R, Roshangar L, Jahanban-Esfahlan R, et al.: Tumor microenvironment complexity and therapeutic implications at a glance. Cell Commun Signal 18: 59, 2020. https://doi.org/10.1186/s12964-020-0530-4

2. Valkenburg KC, de Groot AE, Pienta KJ: Targeting the tumour stroma to improve cancer therapy. Nat Rev Clin Oncol 15: 366-381, 2018. https://doi.org/10.1038/s41571-018-0007-1

3. Kalluri R, Zeisberg M: Fibroblasts in cancer. Nat Rev Cancer 6: 392-401, 2006. https://doi.org/10.1038/nrc1877

4. Liao Z, Tan ZW, Zhu P, Tan NS: Cancer-associated fibroblasts in tumor microenvironment- accomplices in tumor malignancy. Cell Immunol 343: 103729, 2019. https://doi.org/10.1016/j.cellimm.2017.12.003

5. Calon A, Tauriello DV, Batlle E: TGF-beta in CAF-mediated tumor growth and metastasis. Semin Cancer Biol 25: 15-22, 2014.

https://doi.org/10.1016/j.semcancer.2013.12.008 
6. Hofmeister V, Schrama D, Becker JC: Anti-cancer therapies targeting the tumor stroma. Cancer Immunol Immunother 57: 1-17, 2008.

https://doi.org/10.1007/s00262-007-0365-5

7. Bae JY, Kim EK, Yang DH, et al.: Reciprocal interaction between carcinoma-associated fibroblasts and squamous carcinoma cells through interleukin-1 $\alpha$ induces cancer progression. Neoplasia 16: 928-938, 2014. https://doi.org/10.1016/j.neo.2014.09.003

8. Simian M, Hirai Y, Navre M, Werb Z, Lochter A, Bissell MJ: The interplay of matrix metalloproteinases, morphogens and growth factors is necessary for branching of mammary epithelial cells. Development 128: 3117-3131, 2001.

9. Olson OC, Joyce JA: Cysteine cathepsin proteases: regulators of cancer progression and therapeutic response. Nat Rev Cancer 15: 712-729, 2015. https://doi.org/10.1038/nrc4027

10. Leto G, Tumminello FM, Crescimanno M, Flandina C, Gebbia N: Cathepsin D expression levels in nongynecological solid tumors: clinical and therapeutic implications. Clin Exp Metastasis 21: 91-106, 2004. https://doi.org/10.1023/b:clin.0000024740.44602.b7

11. Ashraf Y, Mansouri H, Laurent-Matha V, et al.: Immunotherapy of triple-negative breast cancer with cathepsin D-targeting antibodies. J Immunother Cancer 7: 29, 2019. https://doi.org/10.1186/s40425-019-0498-z

12. Kim DK, Kim EK, Jung DW, Kim J: Cytoskeletal alteration modulates cancer cell invasion through RhoA-YAP signaling in stromal fibroblasts. PLoS One 14: e0214553, 2019. https://doi.org/10.1371/journal.pone.0214553

13. Calvo F, Ege N, Grande-Garcia A, et al.: Mechanotransduction and YAP-dependent matrix remodelling is required for the generation and maintenance of cancerassociated fibroblasts. Nat Cell Biol 15: 637-646, 2013. https://doi.org/10.1038/ncb2756

14. Bhowmick NA, Chytil A, Plieth D, et al.: TGF-beta signaling in fibroblasts modulates the oncogenic potential of adjacent epithelia. Science 303: 848-851, 2004. https://doi.org/10.1126/science.1090922

15. Bruchard M, Mignot G, Derangère V, et al.: Chemotherapytriggered cathepsin $\mathrm{B}$ release in myeloid-derived suppressor cells activates the Nlrp3 inflammasome and promotes tumor growth. Nat Med 19: 57-64, 2013. https://doi.org/10.1038/nm.2999

16. Heylen N, Vincent LM, Devos V, Dubois V, Remacle C, Trouet A: Fibroblasts capture cathepsin D secreted by breast cancer cells: possible role in the regulation of the invasive process. Int J Oncol 20: 761-767, 2002. https://doi.org/10.3892/ijo.20.4.761

17. Wang F, Duan R, Chirgwin J, Safe SH: Transcriptional activation of cathepsin $\mathrm{D}$ gene expression by growth factors. J Mol Endocrinol 24: 193-202, 2000. https://doi.org/10.1677/jme.0.0240193 